\title{
Neuropsychiatric adverse effects of montelukast in children
}

\author{
Pierre Ernst ${ }^{1,2}$ and Geneviève Ernst ${ }^{3}$
}

\begin{abstract}
Affiliations: ${ }^{1}$ McGill University, Montreal, QC, Canada. ${ }^{2}$ Lady Davis Research Institute, Jewish General Hospital, Montreal, QC, Canada. ${ }^{3}$ University of British Columbia, Vancouver, BC, CanadaDept of Pediatrics, BC Children's Hospital, Vancouver, BC, Canada.
\end{abstract}

Correspondence: Pierre Ernst, Centre for Clinical Epidemiology, Lady Davis Research Institute, Jewish General Hospital, 3755 Cote Ste-Catherine, Montreal, Quebec H3T 1E2, Canada.

E-mail: pierre.ernstamcgill.ca

@ERSpublications

Neuropsychiatric ADRs of montelukast are common and difficult to recognise in children http://ow.ly/wlzW30cQn0f

Cite this article as: Ernst P, Ernst G. Neuropsychiatric adverse effects of montelukast in children. Eur Respir J 2017; 50: 1701020 [https://doi.org/10.1183/13993003.01020-2017].

Asthma is the most common chronic disease of childhood. It is associated with significant morbidity and costs including missed time from school and work for children and their parents. For those children $\geqslant 5$ years of age with persistent symptoms or frequent exacerbations, inhaled corticosteroids (ICS) provide an effective and safe treatment, at least at low doses. However, parents are often concerned about their systemic absorption. Common adverse drug reactions with ICS include oropharyngeal thrush and slowing in growth; the latter results, on average, in a $1-\mathrm{cm}$ decrement in adult height [1]. Partly as a result of the fear of adverse events with corticosteroids, ICS may be underprescribed and adherence to sustained treatment with these agents is poor. Furthermore, adolescents may find that one pill a day is less obtrusive and easier to remember. In this context, leukotriene receptor antagonists such as montelukast are attractive. Montelukast, as a daily controller medication, provides efficacy similar, although less potent, than ICS with the possibility of improved adherence such that the overall clinical benefit may be equal [2]. Montelukast is therefore frequently used as the initial controller therapy as well as in addition to low-dose ICS. The latter indication is the one recognised by asthma guidelines [3].

In this issue of the European Respiratory Journal, BENARD et al. [4] examine the frequency and relative risk of neuropsychiatric adverse events in children prescribed montelukast, as compared to ICS, in the real-world setting of a paediatric asthma clinic. Neuropsychiatric adverse events are of particular importance since psychiatric disorders have been found to account for the most important costs associated with comorbidity in asthma [5]. The study was made possible by the systematic collection of clinical information in an electronic medical record; $85 \%$ of patients approached provided parental approval. This information was later linked to a drug prescription database. Children between the ages of 1 and 17 years with a physician diagnosis of asthma were eligible. Parents were contacted by telephone to answer a nonleading standardised questionnaire concerning adverse drug reactions (ADRs) of all types experienced by their children with any asthma medication and then provided with a list of possible neuropsychiatric ADRs. The responses to this telephone interview were then assessed by an expert panel blinded to the asthma therapy received as to whether they were probably or possibly related to the asthma controller therapy alternatively assuming that this therapy was montelukast or ICS.

Of 223 children who initiated montelukast, 106 completed the interview. Responders and nonresponders were roughly comparable except that nonresponders were less likely to be Caucasian. The median age was

Received: May 182017 | Accepted: May 262017

Conflict of interest: None declared

Copyright OERS 2017 
5 years with $75 \%$ being $\leqslant 8$ years old. The primary outcome, the incidence of neuropsychiatric ADRs serious enough to lead to cessation of therapy, was reported by $16 \%$, or $12 \%$ if considering only ADRs definitely or probably related to montelukast. All ADRs were judged to be mild or very mild and only required cessation of therapy. The ADRs reported were irritability, aggressiveness and sleep disturbances. There was no report of suicidal ideation. These adverse events occurred soon after initiation of therapy (median 7 days) and resolved quickly after stopping the medication (median 2 days). The expert panel judged the likelihood that the neuropsychiatric ADR was related to the controller therapy with the same assurance whether they assumed the controller therapy was montelukast or ICS, suggesting that imputability was not affected by pre-conceived notions of the potential adverse effects of montelukast.

When comparing 84 children initiating montelukast to 84 children initiating ICS at a similar time, those initiating montelukast were estimated to be 12 times as likely to experience a neuropsychiatric ADR, although the confidence interval was wide (relative risk 12.0, 95\% CI 1.60-90.2). When looking at individual predictors of adverse reactions to montelukast, the power was low. Only concomitant use of a long-acting $\beta_{2}$-agonist/ICS combination could be significantly related to the risk of an ADR. This, however, probably reflects the severity of asthma, which is itself associated with neuropsychiatric symptoms [6]. There was no significant association between adverse events and polymorphisms of genes associated with the hepatic metabolism of montelukast or its transport across cell membranes, including the blood-brain barrier.

The study was probably prompted by the clinical experience of the investigators as well as spontaneous adverse event reporting that led to a Food and Drug Administration (FDA) advisory in the USA in 2008. Notably, such adverse events were not reported in the initial clinical trials which led to licensing of this medication in children nor in the meta-analysis of these trials [7-9].That neuropsychiatric adverse events were not reported in the paediatric clinical trials of montelukast is surprising and disturbing. Clinical trials cannot be expected to detect rare adverse events, therefore necessitating pharmacovigilance programmes for signal detection and large pharmacoepidemiological studies for confirmation of harm or reassurance of safety [10]. However, here we are dealing with adverse events that appear common, occurring in $>10 \%$ of children. This underscores the highly selected nature of the population targeted for clinical trials. For example, in the study by BENARD et al. [4], 20\% of the children initiating montelukast were labelled as having attention deficit disorder and $16 \%$ of families reported psychological or psychiatric conditions. It is not difficult to understand that these children and families might be less likely to take part in clinical trials. Consequently, the results of clinical trials cannot be applied directly to the patients clinicians see in practice. This limited generalisability is further compounded by good evidence that adverse drug effects are underreported in publications of clinical trial results [11].

There are several implications of the study by Benard et al. [4]. Firstly, clinical trials do not, by themselves, adequately assess the occurrence of adverse effects of medications. It remains the responsibility of the treating physician to have easy access to up-to-date online tools to check for reported adverse events and to engage patients actively to report possible adverse drug effects. At a meeting of the Pediatric Advisory Committee of the FDA held in September 2014, it was suggested that many healthcare professionals were unaware of the neuropsychiatric adverse events related to use of montelukast and that steps to increase awareness were required. Given that neuropsychiatric symptoms in children may be attributed to a wide variety of conditions and possible triggers, it is imperative that knowledge of this common ADR of montelukast be disseminated not only to paediatric respirologists but also to paediatricians and primary care providers. However, physician education alone is unlikely to result in sustained change in prescribing practices. Monitoring for neuropsychiatric ADRs should be integrated into existing asthma guidelines and individual clinics should explore ways to remind physicians to ask families about this important ADR. Moreover, patients should not only be asked about common ADRs at follow-up visits but there also needs to be a system in place for families to report new concerns to their physician as they arise. The transition to electronic medical records in outpatient clinics will provide great opportunities for ongoing ADR surveillance and automated physician alerts. Most importantly, empowering families to report common ADRs on an ongoing basis would allow their physician to adjust their treatment plan sooner and may improve compliance, in addition to overall patient satisfaction.

As for the use of montelukast in children, adverse neuropsychiatric effects are sufficiently common and potentially difficult to recognise in young children to require that parents be informed of these at the initiation of treatment. Notably, the current study included only a small number of adolescents, such that the risk of suicidal behaviour in adolescents reported with montelukast cannot be quantified by the study by BENARD et al. [4]. However, it may be prudent to discuss the possibility of mood changes as well as warning signs for suicidality with both adolescents and their parents when initiating treatment. Finally, it would be helpful to have larger studies that would permit the identification of individual predictors of neuro-psychiatric adverse events related to montelukast. 
References

1 Zhang L, Prietsch SO, Ducharme FM. Inhaled corticosteroids in children with persistent asthma: effects on growth. Evid Based Child Health 2014; 9: 829-930.

2 Price D, Musgrave SD, Shepstone L, et al. Leukotriene antagonists as first-line or add-on asthma-controller therapy. N Engl J Med 2011; 364: 1695-1707.

3 Lougheed MD, Lemiere C, Ducharme FM, et al. Canadian Thoracic Society 2012 guideline update: Diagnosis and management of asthma in preschoolers, children and adults. Can Respir J 2012; 19: 127-164.

4 Benard B, Bastien V, Vinet B, et al. Neuropsychiatric adverse drug reactions in children initiated on montelukast in real-life practice. Eur Respir J 2017; 50: 1700148.

5 Chen W, Lynd LD, FitzGerald JM, et al. Excess medical costs in patients with asthma and the role of comorbidity. Eur Respir J 2016; 48: 1584-1592.

6 Zhang T, Carleton BC, Prosser RJ, et al. The added burden of comorbidity in patients with asthma. J Asthma 2009; 46: 1021-1026.

7 Knorr B, Matz J, Bernstein JA, et al. Montelukast for chronic asthma in 6- to 14-year-old children: a randomized double-blind trial. Pediatric Montelukast Study Group. JAMA 1998; 279: 1181-1186.

8 Bisgaard H, Zielen S, Garcia-Garcia ML, et al. Montelukast reduces asthma exacerbations in 2- to 5-year-old children with intermittent asthma. Am J Respir Crit Care Med 2005; 171: 315-322.

9 Chauhan BF, Ben Salah R, Ducharme FM. Addition of anti-leukotriene agents to inhaled corticosteroids in children with persistent asthma. Cochrane Database Syst Rev 2013; CD009585.

10 Suissa S, Henry D, Caetano P, et al. CNODES: the Canadian Network for Observational Drug Effect Studies. Open Med 2012; 6: e134-e140.

11 Golder S, Loke YK, Wright K, et al. Reporting of adverse events in published and unpublished studies of health care interventions: a systematic review. PLoS Med 2016; 13: e1002127. 